\title{
Antibacterial Treatment of Meticillin-Resistant Staphylococcus Aureus Complicated Skin and Soft Tissue Infections: a Cost and Budget Impact Analysis in Greek Hospitals
}

\author{
Kostas Athanasakis - Ioannis Petrakis - Mark Ollandezos • Christos Tsoulas • \\ Dipen A. Patel $\cdot$ Eleftheria Karampli $\cdot$ John Kyriopoulos
}

To view enhanced content go to www.infectiousdiseases-open.com

Received: July 24, 2014 / Published online: October 7, 2014

(C) The Author(s) 2014. This article is published with open access at Springerlink.com

\section{ABSTRACT}

Introduction: Meticillin-resistant staphylococcus aureus (MRSA) is an important cause of antimicrobial-resistant infections worldwide. Its prevalence remains high in the Greek hospital setting. Complicated skin and soft tissue infections (cSSTIs) due to MRSA are associated with prolonged hospitalization, additional healthcare costs and significant morbidity. The purpose of this study was to conduct a cost analysis and a budget impact analysis relative to different management scenarios for MRSA-cSSTIs from a hospital perspective.

Electronic supplementary material The online version of this article (doi:10.1007/s40121-014-0044-8) contains supplementary material, which is available to authorized users.

K. Athanasakis · M. Ollandezos · E. Karampli ( $₫)$. J. Kyriopoulos

Department of Health Economics, National School of Public Health, 196 Alexandras Ave.,

11521 Athens, Greece

e-mail: ekarabli@esdy.edu.gr

I. Petrakis · C. Tsoulas

Pfizer Hellas, Athens, Greece

D. A. Patel

Pharmerit International, Bethesda, MD, USA
Methods: Equal efficacy was assumed for the pharmacotherapies under evaluation and resource use was elicited via an expert panel of seven local infectious disease specialists. The model was based on a previously published economic model that was adapted for the Greek hospital setting and included a decision tree for the management of hospitalized patients with MRSA-cSSTIs, which simulated costs and outcomes for the duration of hospitalization according to the therapeutic scenario. Inpatient costs consisted of hospitalization, diagnostic/ laboratory testing, physician visits and antibiotic treatment.

Results: Current economic impact of MRSAcSSTIs for the inpatient setting in Greek hospitals was estimated at $€ 29,196,218$. Total per patient cost according to first-line agent was $€ 2,457, € 2,762, € 2,850, € 3,494$ and $€ 3,094$ and mean length of stay was 9.2, 12.5, 10.3, 13.0 and 14.0 days for linezolid, vancomycin, daptomycin, tigecycline, and teicoplanin, respectively. An estimated 10,287 MRSA-cSSTI patients are treated annually in Greek hospitals. Thus, increasing the use of linezolid by $11 \%$ over a 3-year period (current use 19\%; 3 year projection $30 \%$ ), for the management of 
MRSA-cSSTIs, could result in 3-year savings of $€ 896,065$.

Conclusion: Management of MRSA-cSSTI requires intensive resource use; overall healthcare costs differ according to the chosen first-line treatment. In light of considerable budget constraints, development of hospital strategies which facilitate early discharge, such as the introduction of clinical criteria and guidelines for switching from intravenous to oral MRSA-cSSTI therapy, could result in substantial savings for the Greek hospital budget.

Keywords: Antibacterial agents; Complicated skin and soft tissue infection; Cost analysis; Daptomycin; Greek hospitals; Linezolid; Meticillin-resistant Staphylococcus aureus; Teicoplanin; Tigecycline; Vancomycin

\section{INTRODUCTION}

Complicated skin and soft tissue infections (cSSTIs) are among the most common infections treated in the hospital setting $[1,2]$. They represent the more extreme end of the clinical spectrum of SSTIs [3] as they typically involve deep soft tissue and occur in patients with underlying disease, often requiring intravenous (IV) antibiotic therapy and/or surgical intervention [2]. As a result, their treatment incurs high healthcare costs. cSSTIs frequently complicate clinically significant medical conditions such as peripheral arterial disease, diabetes mellitus or renal insufficiency and can also cause complications in immunocompromised hosts [4].

Gram-positive pathogens are the predominant cause of cSSTIs. Staphylococcus aureus ( $S$. aureus) was found to be the most frequently occurring pathogen among hospitalized SSTI patients on a global scale in the SENTRY Antimicrobial Surveillance Program [5], and in a large observational study with 1995 cSSTIs patients hospitalized in Europe [1]. Antibiotic resistance in isolates from SSTIs has increased significantly over time [6-10], but the emergence of meticillin-resistant $S$. aureus (MRSA) is recognized as the greatest resistance concern [5], as it complicates the treatment of cSSTIs, limits therapeutic options and impacts treatment outcomes.

Systemic infections with MRSA are associated with a higher mortality rate, a longer length of stay (LOS) in hospital, and greater healthcare costs than non-MRSA infections [11].

In Greece, where antimicrobial drug resistance rates remain high [12], MRSA prevalence reaches $40 \%$, which is among the highest in Europe [13] and comparable to figures reported for the USA [14]. The increase in MRSA, in part, reflects the changing epidemiology of soft tissue infections as a consequence of the recent dramatic increase in the incidence of community acquired MRSA (CA-MRSA), the pathogen most commonly isolated from SSTIs in many locations in the US [15]. CA-MRSA strains are distinct from hospital-acquired strains, cause mainly skin and soft tissue infections (ranging in severity from furuncles to necrotizing fasciitis), and are associated with the production of virulence factors like the Panton Valentine Leukocidin (PVL) toxin, which may further complicate treatment [16]. In Europe, the prevalence of infections due to CA-MRSA appear to vary across the continent, with Greece reporting one of the highest rates (30\%) of S. aureus infection in outpatients compared to $6-18 \%$ in other Western European countries [16, 17].

Successful management of cSSTI involves prompt recognition, timely surgical debridement or drainage, resuscitation if 
required and appropriate antibiotic therapy [3]. Treatment for MRSA-related cSSTIs may include either IV or per os (PO) pharmaceutical treatment for 7-14 days and in most cases hospitalization is required. Vancomycin (VANCO) has historically been the standard of care for MRSA infections, but adverse effects, the need for IV access and growing resistance, tend to limit its use [7]. Newer antimicrobials such as daptomycin (DAPTO), linezolid (LINE) and telavancin have shown comparable efficacy to VANCO and are recommended in international guidelines [18].

Use of proper antimicrobial treatment is an illustrative example of the tradeoffs between increased pharmaceutical spending and decreased resource use costs. Given the scarcity of economic and human healthcare resources and the fact that the cost of available antimicrobial treatments varies, the purpose of the present study was to identify healthcare resource use and cSSTI total inpatient costs, along with other associated costs for different antibiotic treatments in the Greek hospital setting. A secondary objective was to perform a budget impact analysis for the inpatient treatment of MRSA-cSSTIs.

\section{METHODS}

\section{Model Overview}

A previously published economic model [19] was adapted for the Greek hospital setting, to examine the healthcare resource use and associated economic impact of different management approaches for MRSA-cSSTIs. The model was developed using Microsoft Excel and Visual Basic, and was based on a decision tree for the management of hospitalized patients with MRSA-cSSTIs (Fig. 1), which simulated costs and outcomes for the duration of hospitalization according to different therapeutic scenarios.

The time horizon for the decision tree was a maximum of 14 days including inpatient empirical and first-line treatment where all patients remained hospitalized.

The most common first-line treatment pathways in the management of MRSA-cSSTIs were elicited from a panel of seven local experts (infectious disease specialists), i.e., DAPTO, LINE, teicoplanin (TEICO), tigecycline (TIGE), and VANCO. Cotrimoxazole or tetracyclines were not considered as, according to the expert panel, these are most commonly used for outpatients with mild SSTIs and are not preferred in the management of hospitalized patients with MRSA confirmed cSSTIs.

Efficacy rates for LINE and VANCO were assumed to be equal in terms of clinical success for the length of treatment (89.8\%)—weighted average between LINE clinical success rate (219 out of 239 treated cases) and VANCO clinical success rate (193 out of 220 treated cases), as reported by the non-inferiority study of Itani et al. [20]. For the purpose of the analysis and for simplicity reasons, DAPTO, TIGE and TEICO were also assumed to present equal efficacy rates. Regardless of efficacy assumptions, the adverse event failure rate was calculated as (1success rate) $\times 0.33$, assuming $1 / 3$ of treatment failures are due to adverse events and $2 / 3$ are due to lack of efficacy [11]. Patients not cured after receiving first-line antibiotic treatment or who presented with adverse events were assumed to be cured after receiving secondline therapy. The set of patients who discontinued or failed therapy required additional hospitalization days as per the input of the expert panel. As this study was 


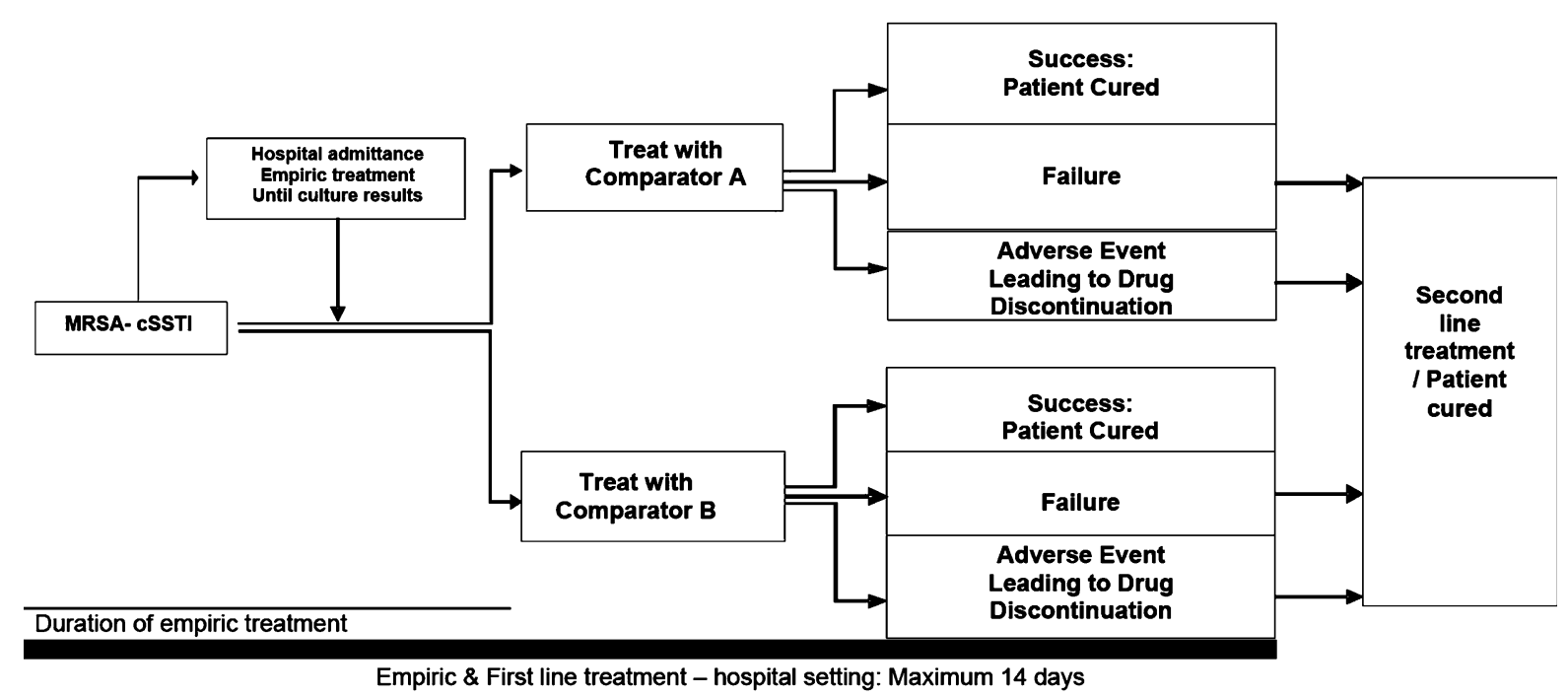

Fig. 1 General model outline for the first-line management of hospitalized MRSA-cSSTI patients. cSSTI complicated skin and soft tissue infections, MRSA meticillin-resistant Staphylococcus aureus

conducted from a hospital perspective, treatment continuation with second-line agents in the outpatient setting was not incorporated into the analysis.

\section{Model Input Data}

Treatment pathways and associated resource use relevant to the local setting were obtained from an expert panel, made up of seven infectious disease specialists. To be included as a member of the expert panel, each participant was required to have long-term, recognized professional experience in treating patients with MRSA-cSSTIs. The panel was varied in terms of level and setting of healthcare provision, i.e., university clinics, NHS hospitals and community clinics, as well as geographic distribution across the Greek mainland.

Data were gathered using a series of closed type questions pertaining to the current management of a "typical" MRSA-cSSTI patient treated in Greece. Resources included hospitalization, hospital procedures, medical visits, laboratory/diagnostic tests and medication associated with MRSA-cSSTIs and its complications. The mean values from all expert responses gathered were used in the study. In view of the lack of relevant data from Greek hospitals, the panel of experts provided information on estimated antibacterial use. The expert panel also provided the information on additional healthcare resource requirements, collectively indicating that drug failures and drug discontinuations due to adverse events resulted in an additional seven and five hospital days, respectively. Moreover, an average per patient-per day hospitalization cost for MRSA-cSSTIs was estimated based on cSSTI diagnosis and diagnosis-related group (DRG) mapping. This was deemed appropriate as several DRG codes were identified by the group of experts as being cSSTI relevant. Continuation of first-line treatment in the outpatient setting was not taken into consideration due to the fact that the study was conducted from a hospital perspective only. 
The resource usage pattern for different treatments (as elicited by the expert panel) is reported in Table 1 . Unit costs for antibiotic agents and inpatient stays are reported in Table 2.

\section{Model Outcomes}

Inpatient costs consisted of hospitalization, diagnostic/laboratory testing, physician visits

Table 1 First-line treatment resource use according to expert panel

\begin{tabular}{|c|c|c|c|c|c|}
\hline \multirow[t]{2}{*}{ Resource usage $^{a}$} & \multicolumn{5}{|c|}{ Mean values reported by the expert panel } \\
\hline & DAPTO & LINE & TEICO & TIGE & VANCO \\
\hline No. of days of treatment ${ }^{\mathrm{b}}$ & 9.8 & 9.2 & 11.5 & 11.7 & 11.8 \\
\hline No. of days in hospital ${ }^{c}$ & 10.3 & 9.2 & 14.0 & 13.0 & 12.5 \\
\hline No of days IV & 9.8 & 6.7 & 12 & 11.7 & 11.8 \\
\hline Average daily dose $(\mathrm{mg})^{\mathrm{d}}$ & 458 & 1,200 & 500 & 133 & 2,000 \\
\hline IV doses per day & 1.0 & 2.0 & 1.0 & 2.0 & 2.0 \\
\hline No. of days oral & 0.0 & 2.5 & 0.0 & 0.0 & 0.0 \\
\hline Oral doses per day & 0.0 & 2.0 & 0.0 & 0.0 & 0.0 \\
\hline Additional no. of days in hospital due to $\mathrm{AE}$ & 5 & 5 & 5 & 5 & 5 \\
\hline Additional no. of days in hospital due to treatment failure & 7 & 7 & 7 & 7 & 7 \\
\hline
\end{tabular}

$A E$ adverse events, DAPTO daptomycin, $I V$ intravenous, LINE linezolid, TEICO teicoplanin, TIGE tigecycline, $V A N C O$ vancomycin

a Values are calculated as means of expert panel feedback

b Excluding the treatment time prior to cultivation results

c Time from hospital admission to discharge

d Estimated for a 'typical' $75 \mathrm{~kg}$ patient

Table 2 Unit cost of inputs

\begin{tabular}{|c|c|c|}
\hline Resource & Input cost & Source/assumption \\
\hline Inpatient cost per day & $€ 189.20$ & $\begin{array}{l}\text { Mean DRG cost for cSSTI, } \\
\text { weighted according to expert panel, } \\
\text { based on diagnosis }[21]\end{array}$ \\
\hline Vancomycin IV $1 \mathrm{~g}$ (empiric-35\%, first line) & $€ 8.92$ & Cheapest generic selected [22] \\
\hline $\begin{array}{l}\text { Other IV empiric (daptomycin-26\%, Linezolid-14\%, } \\
\text { teicoplanin-9\%, Tigecycline-12\%, clindamycin-4\%) }\end{array}$ & $€ 33.89$ & $\begin{array}{l}\text { Average weighted daily cost according to } \\
\text { usage \% in the Greek hospital setting }\end{array}$ \\
\hline Daptomycin IV $500 \mathrm{mg}$ & $€ 87.50$ & [22] \\
\hline Linezolid IV $600 \mathrm{mg}$ & $€ 37.17$ & [22] \\
\hline Linezolid PO tab $600 \mathrm{mg}$ & $€ 32.80$ & [22] \\
\hline Tigecycline IV $50 \mathrm{mg}$ & $€ 37.10$ & [22] \\
\hline Teicoplanin IV $400 \mathrm{mg}$ & $€ 18.41$ & {$[22]$} \\
\hline
\end{tabular}

$D R G$ diagnosis-related group, $I V$ intravenous, $P O$ per os (orally) 
and antibiotic medication. Cost calculations were based on the corresponding tariffs of all services as reported in the official price lists of the National Health System. Hospitalization costs were based upon the current DRG system [21]. Prices for medication were obtained from the Official Price Bulletin [22]. All costs are reported in Euros for the year 2013. The budget impact analysis employed increasing market share for the most cost-saving antibacterial agent over a 3-year period, over the remaining most commonly used antibacterial agents, whose usage was decreased proportionally. Finally, drug price erosion was not taken into account.

\section{Compliance with Ethics Guidelines}

This article does not contain any new studies with human or animal subjects performed by any of the authors.

\section{RESULTS}

\section{Resource Use Pattern}

According to the expert panel, MRSA-cSSTI patients in Greece are 56.6\% males and most of them are over 60 years of age (57.1\%). Patients receive empirical treatment for 2.57 days on average, until a culture-based antibiotic was selected. The panel also concluded that side effects leading to treatment discontinuation resulting from (any) pharmaceutical treatment were reported for $25.7 \%$ of cSSTI-treated patients in the inpatient setting (adverse events failure rate). The average additional LOS due to complications related to pharmaceutical treatment was reported at 4.8 days. The weighted mean DRG reimbursement cost per hospitalization day for the inpatient management of MRSA-cSSTI infections was found to be 189.20 Euros. The proportion of population matching each DRG-cSSTI code was used as weights to calculate mean cost per hospitalization day for the total cSSTI population. The total annual inpatient cost associated with cSSTIs currently in Greece was estimated at $€ 29,196,218$.

According to Table 3, the total per patient cost according to first-line agent was $€ 2,457$, $€ 2,762, € 2,850, € 3,094$ and $€ 3,494$ for LINE, VANCO, DAPTO, TEICO, and TIGE, respectively. LINE as first-line treatment yields the lowest total inpatient cost per patient $(€ 2,457)$ calculated per treatment cycle. This is mostly attributed to the relatively low hospitalization cost per patient $(€ 1,889)$.

The current use of the most common antibacterial agents in the management of cSSTIs, as identified by the expert panel, along with an estimated 3-year gradual increase of the cost-saving antibacterial is presented in Table 4.

Table 5 summarizes the economic impact resulting by gradually increasing LINE use for the treatment of MRSA-cSSTIs from 19\% (current baseline case scenario) to 23\% (year 1 ), to $27 \%$ (year 2) and up to $30 \%$ (year 3). Previously conducted studies estimate that 10,287 MRSA-cSSTI patients are treated annually in Greek hospitals [23, 24]. Inputting these figures into the current study model results in potential savings of $€ 193,291$ (year 1), $€ 354,798$ (year 2) and $€ 347,977$ (year 3) compared to the current baseline case scenario, thus a potential saving of $€ 896,065$ could be made after 3 years.

\section{Sensitivity Analysis}

A series of one-way sensitivity analyses was performed to test the robustness of outcomes 
Table 3 Cost per patient breakdown

\begin{tabular}{|c|c|c|c|c|c|}
\hline \multirow{2}{*}{$\begin{array}{l}\text { Treatment } \\
\text { schedules } \\
\text { (first line) }\end{array}$} & \multicolumn{5}{|c|}{ Total mean inpatient cost/patient (including empiric) $€^{a}$} \\
\hline & $\begin{array}{l}\text { Total inpatient } \\
\text { (pharmaceuticals } \\
\text { and medical) }\end{array}$ & Pharmaceuticals & $\begin{array}{l}\text { Hospitalization } \\
\text { costs }\end{array}$ & $\begin{array}{l}\text { AE management } \\
\text { costs }\end{array}$ & $\begin{array}{l}\text { First-line } \\
\text { failure costs }\end{array}$ \\
\hline LINE & 2,457 & 568 & 1,889 & 58 & 90 \\
\hline VANCO & 2,762 & 249 & 2,513 & 58 & 90 \\
\hline DAPTO & 2,850 & 753 & 2,097 & 58 & 90 \\
\hline TEICO & 3,094 & 300 & 2,796 & 58 & 90 \\
\hline TIGE & 3,494 & 886 & 2,607 & 58 & 90 \\
\hline
\end{tabular}

$A E$ adverse events, DAPTO daptomycin, LINE linezolid, TEICO teicoplanin, TIGE tigecycline, VANCO vancomycin

a Total mean cost per "typical" patient includes medical costs and antibiotic acquisition cost. Medical costs breakdown into cost per day of hospitalization, treatment failure and adverse event costs

Table 4 Current and proposed antibacterial usage estimates

\begin{tabular}{llllll}
\hline & DAPTO & LINE & TEICO & TIGE & VANCO \\
\hline Current cSSTIs usage estimate (\%) & 26 & 19 & 9 & 11 & 35 \\
Proposed usage estimate-year 1 (\%) & 23 & 23 & 10 & 10 & 34 \\
Proposed usage estimate-year 2 (\%) & 19 & 27 & 10 & 10 & 34 \\
Proposed usage estimate-year 3 (\%) & 18 & 30 & 11 & 11 & 30 \\
\hline
\end{tabular}

cSSTI complicated skin and soft tissue infection, DAPTO daptomycin, LINE linezolid, TEICO teicoplanin, TIGE tigecycline, $V A N C O$ vancomycin

a According to expert panel. Teicoplanin and tigecycline estimates considered stable. Any variations are due to rounding up in order to result with assumed $100 \%$ share with the five treatment options

Table 5 Budget impact analysis of the gradual increase of LINE usage in the treatment of meticillin-resistant $S$. aureus complicated skin and soft tissue infections

\begin{tabular}{|c|c|c|c|c|}
\hline & Current cost & Cost after 1 year & Cost after 2 years & Cost after 3 years \\
\hline Inpatient pharmaceuticals & $€ 5,305,213$ & $€ 5,220,891$ & $€ 5,145,002$ & $€ 5,262,737$ \\
\hline Difference & & $-€ 84,322$ & $-€ 160,211$ & $-€ 42,476$ \\
\hline Inpatient medical & $€ 23,891,005$ & $€ 23,782,036$ & $€ 23,696,418$ & $€ 23,585,504$ \\
\hline Difference & & $-€ 108,968$ & $-€ 194,586$ & $-€ 305,501$ \\
\hline Total budget impact & $€ 29,196,218$ & $€ 29,002,927$ & $€ 28,841,420$ & $€ 28,848,241$ \\
\hline Difference & & $-€ 193,291$ & $-€ 354,798$ & $-€ 347,977$ \\
\hline
\end{tabular}

and the impact of individual variables on the results. Table $6[25,26]$ summarizes the results. Parameters with the most significant influence on the results were LINE usage (expanded use led to increased savings), LOS of treatment, and efficacy (success rate) for comparators. Unit prices for LINE and healthcare resources had a lesser impact. 
Table 6 Budget impact results of the univariate sensitivity analyses

Change from baseline value

Total 3-year budget impact for increased use of LINE (€000s)

Baseline case scenario $^{\mathrm{a}}$

$-896.1$

5\% change in LINE use (rise to 24\%)

$15 \%$ change in LINE use (rise to 34\%)

$-1,976.4$

Increase of LINE proportionally to all comparators

$-1,465.3$

Equal LOS assumed for all comparators (14 days)

198.6

Differentiated LOS as reported by Itani et al. [20]

VANCO (mean 8.9 days)

LINE (mean 7.6 days)

$-1,893.6$

Differentiated efficacy (success rate of treatment)

VANCO (88\%)

$-947.1$

as reported by Itani et al. [20]

LINE (92\%)

$10 \%$ increase in the price of LINE

$-777.8$

$10 \%$ decrease in the price of LINE

$-1,006.1$

$10 \%$ increase in all medical costs

$-956.9$

$10 \%$ decrease in all medical costs

Alternative in-hospital per diem cost, as reported by WHO (309.65€), adjusted for
inflation $[25,26]^{\mathrm{d}}$

$-1,284.1$

DAPTO daptomycin, LINE linezolid, LOS length of stay, TEICO teicoplanin, TIGE tigecycline, VANCO vancomycin, WHO World Health Association

${ }^{a}$ LINE use for the treatment of MRSA-cSSTIs increased from 19\% (current use) to 30\% of cases during a 3-year period

${ }^{b}$ Not assumed equal as in the baseline case scenario

${ }^{c}$ Based on the same usage assumptions as in baseline case scenario

${ }^{\mathrm{d}}$ Converted from international dollars

\section{DISCUSSION}

Treatment of cSSTIs due to MRSA has significant cost implications within the hospital setting, as a result of additional LOS and extensive use of appropriate healthcare resources. This study aimed to (a) map the costs associated with inpatient treatment of cSSTIs due to MRSA in Greece, and (b) to analyze costs associated with different antibacterial therapies and the potential budget impact of increasing the usage of the lowest cost agent.

For the baseline case scenario (depicting the current treatment scenario in Greece), LINE was shown to be the most cost-saving first-line antimicrobial agent compared to other treatment options. By extending the scope of its current use (from 19\% of patients to a hypothetical 30\%), the total (aggregate) budget impact was €896,065 over a 3-year period. Although LINE drug acquisition cost approximates the average cost of the remaining available treatments for cSSTI, its use may result in significantly reduced medical costs, namely hospitalization costs, compared to other selected antibiotic treatments. Using LOS data from a large phase IV study [20] for the treatment of documented MRSA-cSSTIs, LINE's budget impact savings were further increased to $€ 1,893,600$ for the 3 -year period 
(Table 6). Clinical data demonstrates 100\% bioavailability [27] of the oral LINE formulation, thus allowing continuation of therapy in both the inpatient and outpatient setting when clinically appropriate and without dosage adjustment.

In addition to reducing LOS and healthcare costs, the Infectious Diseases Society of America and the Society for Healthcare Epidemiology of America Guidelines suggest that conversion from parenteral to oral therapy using antimicrobials with excellent bioavailability, when the patient's condition allows, can reduce complications that may arise as a result of IV access. An antimicrobial management program which outlines clinical criteria and guidelines for oral switch could play an important role in safeguarding the balance between incremental antimicrobial acquisition costs and the development of resistance at an institutional level [28].

Hospitalization has been recognized within international literature as the main cost driver in the treatment of MRSA infections, comprising up to $81 \%$ of total treatment costs and making early discharge a reasonable target for savings [29, 30]. A recent retrospective chart review in 12 European countries [26] has shown that by applying early discharge criteria in MRSA-cSSTIs more than one-third of hospitalized patients in usual clinical practice could be discharged from the hospital (37.9\% overall and $41.1 \%$ for Greece with a mean \pm SD potential LOS reduction of $6.2 \pm 8.2$ and $7.5 \pm 10.6$ days, respectively).

A treatment strategy that would focus on early discharge followed by outpatient parenteral treatment (OPAT) use or use of orally available antimicrobials could be of benefit. However, OPAT treatment is rather costly and is not commonly used in the Greek NHS setting [31].
As with every study of this kind, the outcomes must be interpreted in light of a number of limitations. First of all, the study design assumes equal effectiveness among the different treatment schedules. This probably leads to conservative estimations, considering that data from a recent systematic meta-analysis of 14 studies of six antibiotics $(n=1,840)$ suggested differences in efficacy of different MRSA-cSSTI pharmaceutical treatments [32], mostly in favor of LINE. The assumption that all patients are cured after receiving second-line treatment and that no patients died represents a simplification of reality. The majority of studies in cSSTIs do report high clinical success and very low mortality rates [15] which are similar for patients treated with LINE or VANCO [20], however, second-line efficacy data are lacking. All model assumptions were based on a previously published model [19] and were considered reasonable by the panel of experts. Moreover, the option of outpatient oral and IV treatment continuation was not taken into consideration as this falls beyond the scope of the study (hospital setting). However, this could have a substantial impact on total cost of treatment [33], especially from an overall healthcare system perspective. Recent evidence shows that the administration of antibacterial treatment administered on an outpatient basis can reduce per patient costs up to 30\% [19].

Another limitation to be considered is the lack of Greek-specific pharmaceutical use and MRSAcSSTI prevalence data. The use of an expert panel approach implies a level of uncertainty with respect to the data acquisition process [34]. Furthermore, results may not be fully transferable to other treatment settings, as significant variation exists in clinical practice [31]. The actual magnitude of this uncertainty is very difficult to quantify. However, the multidisciplinary make-up of the team of 
experts and the consistency of estimations produced by expert panels in Greece, as reported by previous research [35] enhance the robustness of results. Moreover, recently published data on patients' age and sex, mean LOS and IV use in Greece from a retrospective study in 12 European countries [31] are consistent with the results of the expert panel.

In actual terms, the economic benefits of LINE are heavily based on its ability to reduce LOS and associated hospital costs. This was evident in the case where equal LOS was hypothesized for all comparators, which resulted in VANCO being the least costly choice. Newer agents have been shown to reduce LOS and thus may offset (partially or fully) the costs of medication.

Finally, the use of the recently introduced DRGs costing system leads to an underestimation of the relevant costs. This is because the current DRG price lists in Greece do not include staff costs. This is likely to result in an underestimation of the actual cost of treating cSSTIs due to MRSA, especially within the hospital setting. It has to be noted that the average hospitalization cost per day for cSSTIs based on the DRG reimbursement system was found to be approximately half the respective cost compared to recently published literature in the Greek setting [31] and the World Health Organization for inpatient cost per day (309.65 adjusted for inflation in 2012) $[25,26]$. Furthermore, as with most pharmacoeconomic studies, drug acquisition costs used in the cost calculations lack consideration for price variation over time.

Notwithstanding its limitations, this study is an illustrative example of the importance of global hospital budget, where higher drug acquisition costs would be offset by the marked reduction in hospital and fixed costs due to early discharge.

\section{CONCLUSION}

According to current clinical practice, as depicted by the panel of experts in the present study, LINE is associated with the lowest total costs in the management of the "typical" MRSAcSSTI patient. The lower costs for patients treated with LINE can be attributed to a switch to oral therapy and earlier hospital discharge. International experience shows that early discharge, when certain criteria are met, can reduce healthcare costs without compromising quality of care. In light of considerable hospital budget constraints, the development of hospital strategies to facilitate early discharge of patients, for example by introducing clinical criteria and guidelines for switching to oral treatments for MRSA-cSSTIs, could result in substantial savings for the Greek global hospital budget.

\section{ACKNOWLEDGMENTS}

All named authors meet the ICMJE criteria for authorship for this manuscript, take responsibility for the integrity of the work as a whole, and have given final approval to the version to be published. Financial support for this study and article processing charges were provided by Pfizer Hellas, Athens, Greece.

Conflict of interest. C. Tsoulas is an employee of Pfizer Hellas and holds stock in Pfizer Inc. I. Petrakis is a former employee of Pfizer Hellas. K. Athanasakis reports research funding from various pharmaceutical companies outside the submitted work. E. Karampli reports research funding from various pharmaceutical companies outside the submitted work. M. Ollandezos reports research funds from various pharmaceutical companies 
outside the submitted work. J. Kyriopoulos reports research funding from various pharmaceutical companies outside the submitted work. D.A. Patel is an employee of Pharmerit, and Pharmerit received research funding from Pfizer Inc to develop the model. No funding was provided to the authors for the manuscript development. All authors had full access to all of the data in this study and take complete responsibility for the integrity of the data and accuracy of the data analysis.

Compliance with ethics guidelines. This article does not contain any new studies with human or animal subjects performed by any of the authors.

Open Access. This article is distributed under the terms of the Creative Commons Attribution Noncommercial License which permits any noncommercial use, distribution, and reproduction in any medium, provided the original author(s) and the source are credited.

\section{REFERENCES}

1. Garau J, Ostermann H, Medina J, et al. Current management of patients hospitalized with complicated skin and soft tissue infections across Europe (2010-2011): assessment of clinical practice patterns and real-life effectiveness of antibiotics from the REACH study. Clin Microbiol Infect. 2013;19(9):E377-85.

2. Zervos MJ, Freeman K, Vo L, et al. Epidemiology and outcomes of complicated skin and soft tissue infections in hospitalized patients. J Clin Microbiol. 2012;50(2):238-45.

3. Dryden MS. Complicated skin and soft tissue infection. $\mathrm{J}$ Antimicrob Chemother. 2010;65(s3):iii35-44.

4. Dryden M. Complicated skin and soft tissue infections caused by methicillin-resistant Staphylococcus aureus: epidemiology, risk factors, and presentation. Surg Infect (Larchmt). 2008;9(s1):s3-10.
5. Moet GJ, Jones RN, Biedenbach DJ, Stilwell MG, Fritsche TR. Contemporary causes of skin and soft tissue infections in North America, Latin America, and Europe: Report from the SENTRY Antimicrobial Surveillance Program (1998-2004). Diagn Microbiol Infect Dis. 2007;57(1):7-13.

6. Stryjewski ME, Chambers HF. Skin and soft-tissue infections caused by community-acquired methicillin-resistant Staphylococcus aureus. Clin Infect Dis. 2008;46(s5):S368-77.

7. Sharpe JN, Shively EH, Polk HC Jr. Clinical and economic outcomes of oral linezolid versus intravenous vancomycin in the treatment of MRSA-complicated, lower-extremity skin and softtissue infections caused by methicillin-resistant Staphylococcus aureus. Am J Surg. 2005;189(4):425-8.

8. White B, Seaton RA. Complicated skin and soft tissue infections: literature review of evidence for and experience with daptomycin. Infect Drug Resist. 2011;4:115-27.

9. Sevinç F, Prins JM, Koopmans RP, et al. Early switch from intravenous to oral antibiotics: guidelines and implementation in a large teaching hospital. J Antimicrob Chemother. 1999;43(4):601-6.

10. Laing RB, Mackenzie AR, Shaw H, Gould IM, Douglas JG. The effect of intravenous-to-oral switch guidelines on the use of parenteral antimicrobials in medical wards. J Antimicrob Chemother. 1998;42(1):107-11.

11. Itani KMF, Merchant S, Lin S-J, Akhras K, Alandete JC, Hatoum HT. Outcomes and management costs in patients hospitalized for skin and skin-structure infections. Am J Infect Control. 2011;39(1):42-9.

12. Miyakis S, Pefanis A, Tsakris A. The challenges of antimicrobial drug resistance in Greece. Clin Infect Dis. 2011;53(2):177-84.

13. European Centre for Disease Prevention and Control. Antimicrobial resistance surveillance in Europe 2011 [Internet]. Annual Report of the European Antimicrobial Resistance Surveillance Network (EARS-Net). Stockholm: ECDC; 2012. Available from: http://www.ecdc.europa.eu/en/ publications/publications/antimicrobial-resistancesurveillance-europe-2011.pdf. Accessed 21 May 2013.

14. Otter JA, French GL. Molecular epidemiology of community-associated meticillin-resistant Staphylococcus aureus in Europe. Lancet Infect Dis. 2010;10(4):227-39.

15. May AK, Stafford RE, Bulger EM, et al. Treatment of complicated skin and soft tissue infections. Surg Infect (Larchmt). 2009;10(5):467-99. 
16. Köck R, Becker K, Cookson B, et al. Meticillinresistant Staphylococcus aureus (MRSA): burden of disease and control challenges in Europe. Euro Surveill. 2010;15(41):19688.

17. Vourli S, Vagiakou H, Ganteris G, et al. High rates of community-acquired, Panton-Valentine leukocidin (PVL)-positive methicillin-resistant $S$. aureus (MRSA) infections in adult outpatients in Greece. Euro Surveill. 2009;14(2). (pii: 19089).

18. Liu C, Bayer A, Cosgrove SE, et al. Clinical practice guidelines by the infectious diseases society of america for the treatment of methicillin-resistant Staphylococcus Aureus infections in adults and children. Clin Infect Dis. 2011;52(3):285-92.

19. Stephens JM, Gao X, Patel DA, Verheggen BG, Shelbaya A, Haider S. Economic burden of inpatient and outpatient antibiotic treatment for methicillinresistant Staphylococcus aureus complicated skin and soft-tissue infections: a comparison of linezolid, vancomycin, and daptomycin. Clinicoecon Outcomes Res. 2013;5:447-57.

20. Itani KMF, Dryden MS, Bhattacharyya H, Kunkel MJ, Baruch AM, Weigelt JA. Efficacy and safety of linezolid versus vancomycin for the treatment of complicated skin and soft-tissue infections proven to be caused by methicillin-resistant Staphylococcus aureus. Am J Surg. 2010;199(6):804-16.

21. Ministerial Decision $\Upsilon 4 \alpha /$ oıк.13740. Greek Government Gazette No 940/B. 2012 [published Mar 27, 2012]. Available from: www.et.gr. [In Greek]. Accessed 31 Jan 2013.

22. Ministry of Health. Price bulletings. Athens: Ministry of Health; 2013. Available from: http:// www.moh.gov.gr/articles/times- farmakwn/deltia-ti mwn. Accessed 31 Oct 2013.

23. McCaig LF, McDonald LC, Mandal S, Jernigan DB. Staphylococcus aureus-associated skin and soft tissue infections in ambulatory care. Emerg Infect Dis. 2006;12(11):1715-23.

24. Jacobus C, Lindsell C, Leach S, Fermann G, Kressel A, Rue L. Prevalence and demographics of methicillin resistant Staphylococcus aureus in culturable skin and soft tissue infections in an urban emergency department. BMC Emerg Med. 2007;7:19.

25. WHO-CHOICE 2013. CHOosing Interventions that are Cost Effective (WHO-CHOICE). Countryspecific unit costs [Internet]. Geneva: World Health Organization; 2013 [updated 2011 July]. Available from: http://www.who.int/choice/country/ country_specific/en/. Accessed 31 Oct 2013.
26. Nathwani D, Eckmann C, Lawson W, et al. Pan-European early switch/early discharge opportunities exist for hospitalized patients with methicillin-resistant Staphylococcus aureus complicated skin and soft tissue infections. Clin Microbiol Infect. 2014;. doi:10.1111/1469-0691. 12632 [Epub ahead of print].

27. Dryden MS. Linezolid pharmacokinetics and pharmacodynamics in clinical treatment. J Antimicrob Chemother. 2011;66(s4):iv7-15.

28. Dellit TH, Owens RC, McGowan JE, et al. Infectious diseases society of America and the society for healthcare epidemiology of America guidelines for developing an institutional program to enhance antimicrobial stewardship. Clin Infect Dis. 2007;44(2):159-77.

29. Goetghebeur M, Landry PA, Han D, Vicente C. Meticillin-resistant Staphylococcus aureus: a public health issue with economic consequences. Can J Infect Dis Med Microbiol. 2007;18(1):27-34.

30. Nathwani D. Impact of meticillin-resistant Staphylococcus aureus infections on key health economic outcomes: does reducing the length of hospital stay matter? J Antimicrob Chemother. 2003;51(s2):ii37-44.

31. Eckmann C, Lawson W, Nathwani D, et al. Antibiotic treatment patterns across Europe in patients with complicated skin and soft-tissue infections due to meticillin-resistant Staphylococcus aureus: a plea for implementation of early switch and early discharge criteria. Int $\mathrm{J}$ Antimicrob Agents. 2014;44(1):56-64.

32. Logman JFS, Stephens J, Heeg B, et al. Comparative effectiveness of antibiotics for the treatment of MRSA complicated skin and soft tissue infections. Curr Med Res Opin. 2010;26(7):1565-78.

33. Seaton RA, Johal S, Coia JE, Reid N, Cooper S, Jones BL. Economic evaluation of treatment for MRSA complicated skin and soft tissue infections in Glasgow hospitals. Eur J Clin Microbiol Infect Dis. 2014;33(3):305-11.

34. Evans C. The use of consensus methods and expert panels in pharmacoeconomic studies. Pharmacoeconomics. 1997;12(2 Pt 1):121-9.

35. Athanasakis K, Ollandezos M, Angeli A, Gregoriou A, Geitona M, Kyriopoulos J. Estimating the direct cost of type 2 diabetes in Greece: the effects of blood glucose regulation on patient cost. Diabet Med. 2010;27(6):679-84. 